\title{
Formation of Nicotinamide Ribose Diphosphate Ribose, a New Metabolite of the NAD Pathway, by Growing Mycelium of Aspergillus niger ${ }^{\dagger}$
}

\author{
Masaaki KUwAHARA \\ Department of Food Science, Kagawa University, Miki-cho, Kagawa \\ Received February 18, 1976
}

\begin{abstract}
A new step of NAD metabolism was shown in Aspergillus niger. Radioactive nicotinic acid and nicotinamide were incorporated into nicotinamide ribose diphosphate ribose (NAmRDPR), which had been isolated from the culture filtrate. Its content in the culture medium increased with an increase of culture time, and this compound was proved to be a terminal metabolite in the NAD pathway. The experimental results also showed that the PreissHandler pathway and the NAD cycling system function in the NAD biosynthesis in $A$. niger. A part of the radioactive precursors was also incorporated into an unknown compound.
\end{abstract}

The Preiss-Handler pathway ${ }^{1)}$ participates in NAD synthesis from both exogenous and endogenous nicotinamide or nicotinic acid, and the presence of this pathway has been demonstrated in various organisms. ${ }^{2)}$ It is well established that the first step of this pathway involves deamidation of nicotinamide to nicotinic acid by nicotinamidase. ${ }^{3)}$ Nicotinic acid is successively converted to NaMN and deamido-NAD and finally to NAD. The hydrolysis of NAD at the nicotinamideribose bond by NADase gives nicotinamide, that is recycled to NAD via the Preiss-Handler pathway. ${ }^{4)}$ Nicotinamide-dependent pathway, through which nicotinamide is incorporated into NAD via NMN without being deamidated to nicotinic acid, has been demonstrated for animal tissues. ${ }^{5,6)}$ Of micoorganisms, Lactobacillus fructosus exceptionally possesses nicotinamide-dependent pathway. This organism lacks nicotinamidase, but has NMN pyrophophorylase activity. ${ }^{7)}$ The authors indicated in a previous report ${ }^{8}$ the production of nicotinamide ribose diphosphate ribose (NAmRDPR) in the degradation of the adenine-ribose bond of NAD by a nucleosidase-type enzyme of Aspergillus niger. This paper deals with the formation of NAmRDPR

$\dagger$ Metabolism of NAD in Aspergillus niger. Part II. See reference 8) for Part I. from precursor nicotinic acid or nicotinamide by growing mycelium of $A$. niger and suggests that this compound is a new metabolite of the Preiss-Handler pathway.

\section{MATERIALS AND METHODS}

Materials. $\quad\left[7 .{ }^{14} \mathrm{C}\right]$ Nicotinamide $(61 \mathrm{mCi}$ permmole) and $\left[7-{ }^{14} \mathrm{C}\right]$ nicotinic acid $(60 \mathrm{mCi}$ per mmole) were obtained from Ammersham. Deamido-NAD was prepared by the method of Hondo et al.$^{8)}$ using pig brain NADase (Boehringer). NaMN was prepared by cleaving deamido-NAD with snake venom phosphodiesterase (Boehringer) and purified by paper chromatography in the isobutyric acid-ammonia-water system. Nicotinamide ribose was obtained by degradation of $5^{\prime}$-NMN with calf intestine alkaline phosphatase (Boehringer). NAmRDPR was prepared by the method reported previously. ${ }^{8)}$

Culture of $A$. niger in the radioactive media. A. niger (AKU 3302) was grown in the medium containing $5 \%$ glucose, $0.5 \%$ peptone, $0.2 \% \mathrm{KH}_{2} \mathrm{PO}_{4}, 0.2 \%\left(\mathrm{NH}_{4}\right)_{2}$ $\mathrm{SO}_{4}, 0.05 \% \mathrm{MgSO}_{4} \cdot 7 \mathrm{H}_{2} \mathrm{O}$ and a radioactive precursor. The medium was adjusted to $\mathrm{pH} 6.0$ with $2 \mathrm{~N} \mathrm{HCl}$. In ordinary experiments, $1.25 \times 10^{-7} \mathrm{Ci}$ of radioactive nicotinic acid or nicotinamide was added to $6 \mathrm{ml}$ of the medium in a test tube. One loopful of $A$. niger

The abbreviations used in this report are: NAD, Nicotinamide adenine dinucleotide; NADP, Nicotinamide adenine dinucleotide phosphate; NMN, Nicotinamide mononucleotide; Deamido-NAD, Nicotinic acid adenine dinucleotide; $\mathrm{NaMN}$, Nicotinic acid mononucleotide; NAmR, Nicotinamide ribose. 
mycelium was inoculated into the medium and cultured with shaking at $28^{\circ} \mathrm{C}$. At the end of culture, mycelium was harvested by filtration with a Sartrius membrane filter. After washing with cold water and subsequent removal of excess moisture on mycelia by a filter paper, the mycelial mat was cut into small pieces and extracted with $1.0 \sim 1.5 \mathrm{ml}$ of $2 \%$ PCA for $30 \mathrm{~min}$ in the cold. The cell debris was removed by centrifugation. The supernatant solution was neutralized with solid $\mathrm{KHCO}_{3}$ or $2 \mathrm{~N} \mathrm{KOH}$ and the precipitate of $\mathrm{KClO}_{3}$ produced was removed by centrifugation. The culture filtrate and the mycelial extract obtained were subjected to chromatography.

Separation of intermediates and measurement of radioactivity. The culture filtrate or the mycelial extract $(30$ or $50 \mu \mathrm{l}$ ) was spotted on Whatman $3 \mathrm{MM}$ paper strip and chromatographed with reference nicotinic acid derivatives using isobutyric acid-ammoniawater $(66: 1.7: 33$, solvent $\mathrm{A}),{ }^{10)} 1 \mathrm{M}$ ammonium acetate (pH 5.0)-ethanol ( $3: 7$, solvent $B)^{11)}$ and butanol saturated with $3 \%$ ammonia (solvent $C)^{12)}$ as the developing solvents. Each chromatogram was scanned in the radiochromatographic scanner (Aloka Model TRM1B) and the radioactive components were located by comparing with the peaks of scans produced with the location of reference nicotinic acid derivatives detected with UV lamp. The chromatogram was then cut into pieces corresponding to each derivative, taken into scintillation vials and analyzed for radioactivity in a liquid scintillation spectrometer (Hitachi-Horiba) using a $0.4 \%$ solution of 2, 5-bis [2-(5-tert-butylbenzoxazolyl)] thiophene in toluene as a scintillation fluid. Column chromatography was also examined for the separation of radioactive intermediates. The culture filtrate into which reference nicotinic acid derivatives were added was applied onto a Dowex $1 \times 2$ (formate) column and the column was eluted with formic acid in a stepwise manner. Elution of nicotinic acid derivatives were detected by measuring the optical absorbance of each eluate fraction at $260 \mathrm{~nm}$. One-ml aliquot of each fraction was taken into a vial and radioactivity was measured in the liquid scintillation spectrometer using PCS (Ammersham/Searle) as a scintillation fluid.

\section{RESULTS}

\section{Excretion of nicotinamide ribose diphosphate} ribose by A. niger

$A$. niger (AKU 3302) was grown in the medium containing nicotinic acid- or nicotinamide $-{ }^{14} \mathrm{C}$ and the metabolites in the culture filtrate were separated by paper chromatography using the two solvent systems as described above. Figures 1 and 2 show that

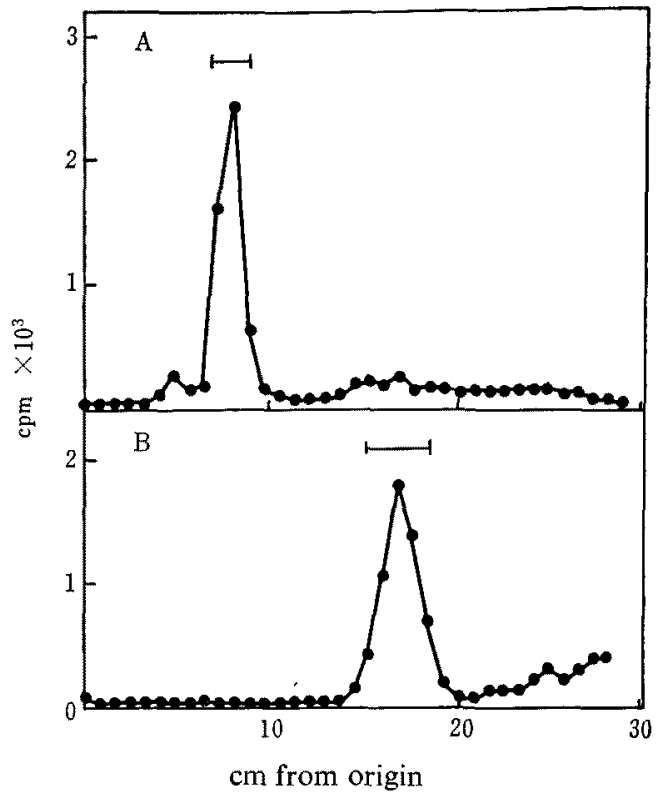

Frg. 1. Separation of Radioactive NAmRDPR in the Filtrate of Nicotinic Acid-added Culture.

A. niger was grown for 3 days. A $50-\mu 1$ aliquot of the culture filtrate was developed in two solvent systems. The chromatogram was cut into $8-\mathrm{mm}$ strips and the radioactivity was counted.

A Chromatogram developed in solvent $A$.

$B$ Chromatogram developed in solvent $B$.

The bar indicates the position of marker NAmRDPR. The positions of other marker compounds are not shown in the figure.

NAmRDPR was the major metabolite in the culture filtrate when either nicotinic acid or nicotinamide served as precursor. On the chromatogram developed in solvent A, NAmRDPR was separated from other nicotinic acid derivatives and the majority of the recorded radioactivity was detected on NAmRDPR. With solvent B, although NAmRDPR ran with NMN and NaMN, the radioactivity attributable to those of NMN and NaMN was negligibly low. A low level of radioactivity was detected near the solvent front with solvent $\mathrm{B}$ and the radioactivity was attributable to those of nicotinic acid and nicotinamide. With solvent $A$, the spot of nicotinic acid was rather broad and no clear peak of radioactivity was observed. Thus, the radioactivity level of NAD and other possible intermediates of NAD metabolism in the culture filtrate was concluded to be low. 


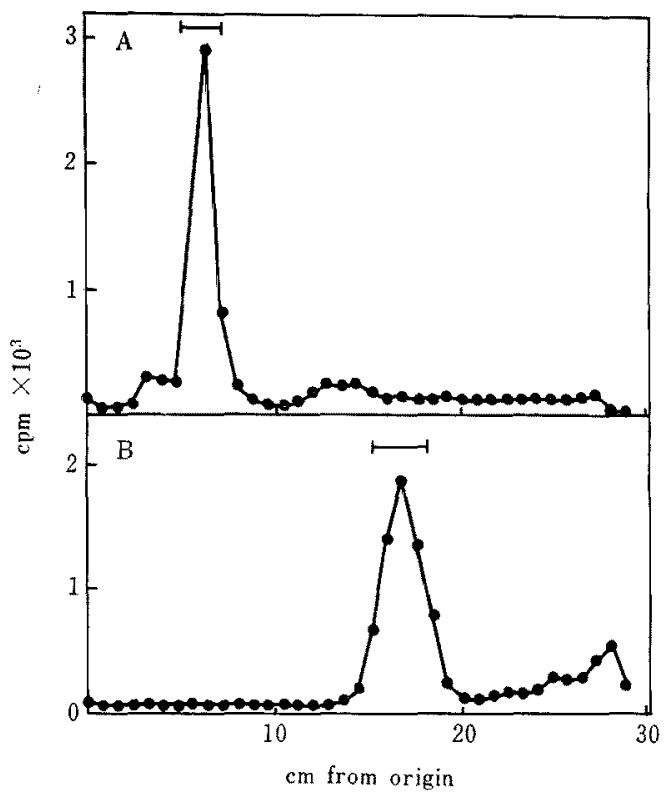

FIG. 2. Separation of Radioactive NAmRDPR in the Filtrate of Nicotinamide-added Culture.

A. niger was grown for 3 days. Analytical methods were the same as that in Fig. 1.

A Chromatogram developed in solvent $A$.

B Chromatogram developed in solvent B.

\section{Separation of radioactive NAmRDPR by column chromatography}

Three 5-day cultures in nicotinic acid- ${ }^{14} \mathrm{C}$ containing medium in test tubes were combined and the culture filtrate was obtained. To $13.5 \mathrm{ml}$ of the filtrate was added $1.5 \mathrm{ml}$ of $20 \%$ PCA. After centrifugation, the supernatant solution was neutralized with $1.5 \mathrm{ml}$ of $4 \mathrm{~N}$ $\mathrm{KOH}$ and the precipitate was centrifuged off. The supernatant solution was added with reference derivatives of nicotinic acid and applied to a column of Dowex $1 \times 2$ (formate form, $1.2 \mathrm{~cm} \times 27 \mathrm{~cm}$ ). The column was eluted with formic acid in a stepwise manner. Fractions in which the derivative was eluted were collected and concentrated by a rotary evaporator. Each concentrate was subjected to paper chromatography and the component was identified by comparing its $R f$ value with that of a reference derivative. The elution pattern is shown in Fig. 3. Adsorption of substances to the resin is thought to be influenced by the inorganic salts in the culture

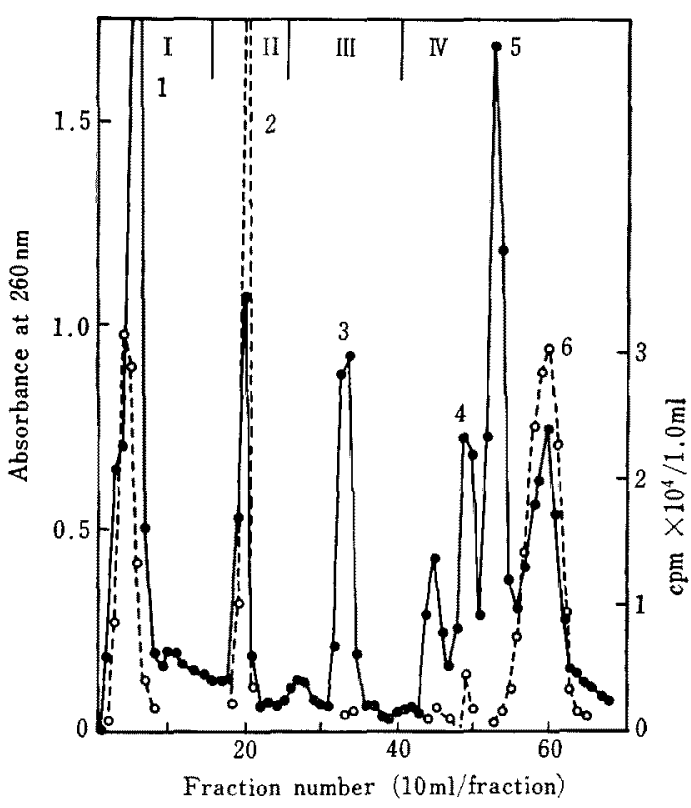

FIG. 3. Separation of NAmRDPR in the Nicotinic Acid-added Medium by Column Chromatography.

Formic acid at the following concentrations was used: (I), water; (II) $0.05 \mathrm{~N}$; (III), $0.2 \mathrm{~N}$; (IV), $2 \mathrm{~N}$.

One-ml aliquot of each fraction was taken into a vial and dissolved in $5 \mathrm{ml}$ of PCS and the radioactivity was counted. Peak 1; nicotinamide-unadsorbed substances, peak 2; nicotinic acid $+\mathrm{NMN}+\mathrm{NAmR}$, peak 3; NAD, peak 4; NaMN, peak 5; deamidoNAD, peak 6; NAmRDPR.

- - absorbance at $260 \mathrm{~nm} ; 0--0$, radioactivity.

medium, which remained in the charged sample solution. Thereby, some portion of the radioactive substances was not adsorbed to the resin column which had been washed with water. Unknown metabolites which could not clearly be detected by paper chromatography may be present in the water fraction. Thus, Fraction 1 contained unadsorbed substances as well as nicotinamide. Radioactivity in Fraction 2 was mainly of nicotinic acid and NMN. NAmRDPR was eluted with $2 \mathrm{~N}$ formic acid in Fraction 6. This fraction was evaporated to dryness and the residue was dissolved in $0.2 \mathrm{ml}$ of water. Paper chromatography of the solution followed by the liquid scintillation counting of the chromatogram showed the radioactive NAmRDPR pure. 


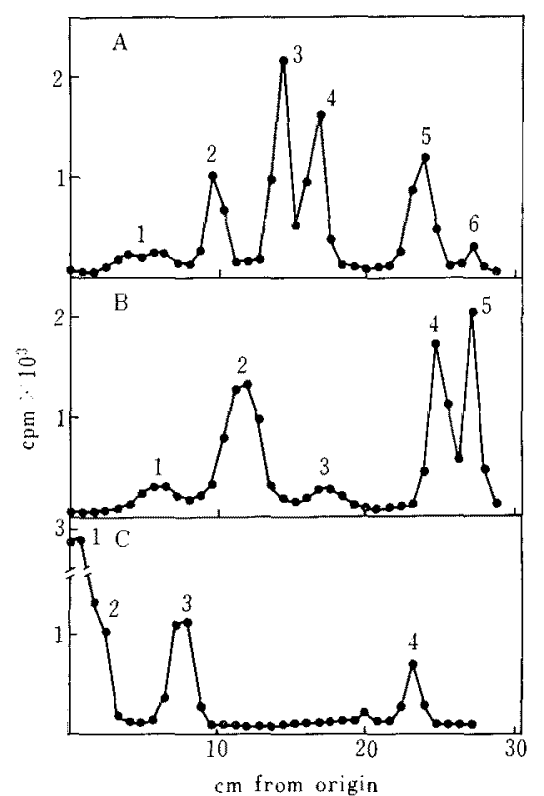

Fig. 4. Separation of the Metabolites in the Acid Extract of Nicotinic Acid-Grown Mycelium.

A. niger was grown in the nicotinic acid-added medium for 3 days. The mycelial mat $(76 \mathrm{mg})$ was extracted with $1.5 \mathrm{ml}$ of $2 \% \mathrm{PCA}$ and the extract was neutralized with solid $\mathrm{KHCO}_{3}$. A $50-\mu$ l aliquot of the supernatant solution was developed in three solvent systems. The chromatogram was cut into 8-mm strips and the radioactivity was counted.

\section{A Chromatogram developed in solvent A.}

Peak 1; NAmRDPR, peak 2; NADP + NaMN+ deamido-NAD, peak 3; NAD $+\mathrm{NMN}$, peak 4; unknown compound, ${ }^{*}$ peak 5: nicotinic acid, peak 6; nicotinamide.

B Chromatogram developed in solvent $\mathbf{B}$.

Peak 1; NADP, peak 2; NAD + deamido-NAD, peak 3; NMN+NaMN+NAmRDPR, peak 4; unknown compound, ${ }^{*}$ peak 5 ; nicotinic acid +nicotinamide.

C Chromatogram developed in solvent $\mathrm{C}$

Peak 1; a mixture of nucleotides, peak 2; unknown compound,* peak 3 ; nicotinic acid, peak 4 ; nicotinamide.

* Nicotinic acid-N-oxide was used as a tentative marker of the unknown compound.

\section{Incorporation of radioactive precursors into} metabolites in cell extract

Figures 4 and 5 show the distribution of radioactivity in mycelia when $A$. niger was grown in radioactive precursors. In the extract of nicotinic acid- ${ }^{14} \mathrm{C}$ grown mycelium (Fig. 4), six peaks of radioactivity were detected on the chromatogram in solvent A. A high level of radioactivity was found on NAD in

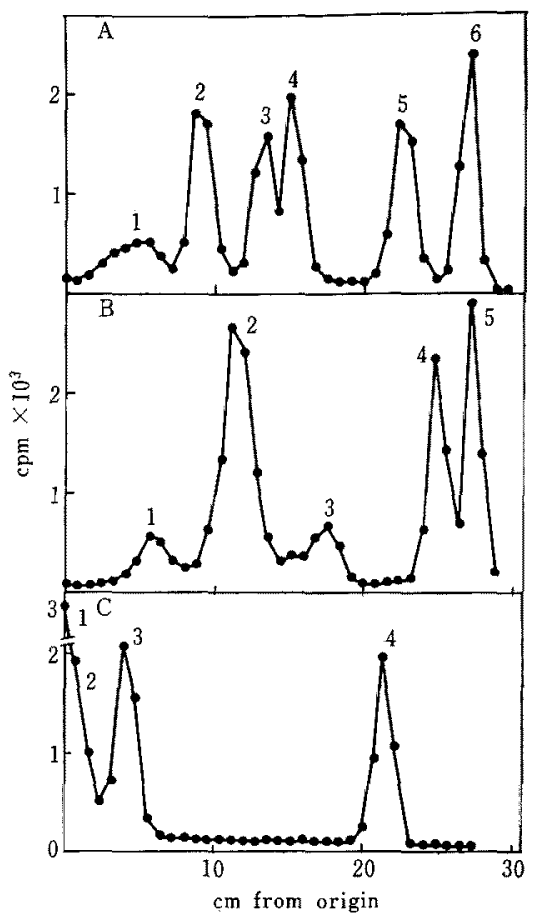

FIG. 5. Separation of the Metabolites in the Acid Extract of Nicotinamide-Grown Mycelium.

$A$. niger was grown in the nicotinamide-added medium for 3 days. The mycelial mat $(136 \mathrm{mg})$ was extracted with $1.5 \mathrm{ml}$ of $2 \% \mathrm{PCA}$, and the extract was neutralized with solid $\mathrm{KHCO}_{3}$. A $50-\mu$ l aliquot of the supernatant solution was subjected to paper chromatography. Analytical methods were the same as that in Fig. 4.

A Chromatogram developed in solvent $A$.

B Chromatogram developed in solvent B.

C Chromatogram developed in solvent $\mathrm{C}$.

the peak 3 area. Although NAD and NMN were developed together in this area, contribution of radioactivity in NMN was apparently low, because the radioactivity of the peak 3 area in solvent $B$, in which NMN was developed separately from NAD, was low. It is thought that radioactivity of peak 2 in solvent $A$, in which NADP, deamido-NAD and NaMN were developed together, was mainly of deamido-NAD, as the chromatographic pattern in solvent $B$ showed that the amount of activity attributable to NADP and NaMN, which ran respectively to peaks 1 and 3 , was low. Presence of radioactivity in the nicotinic acid and nicotinamide areas reflects the NADdegrading activity of $A$. niger and the existence 


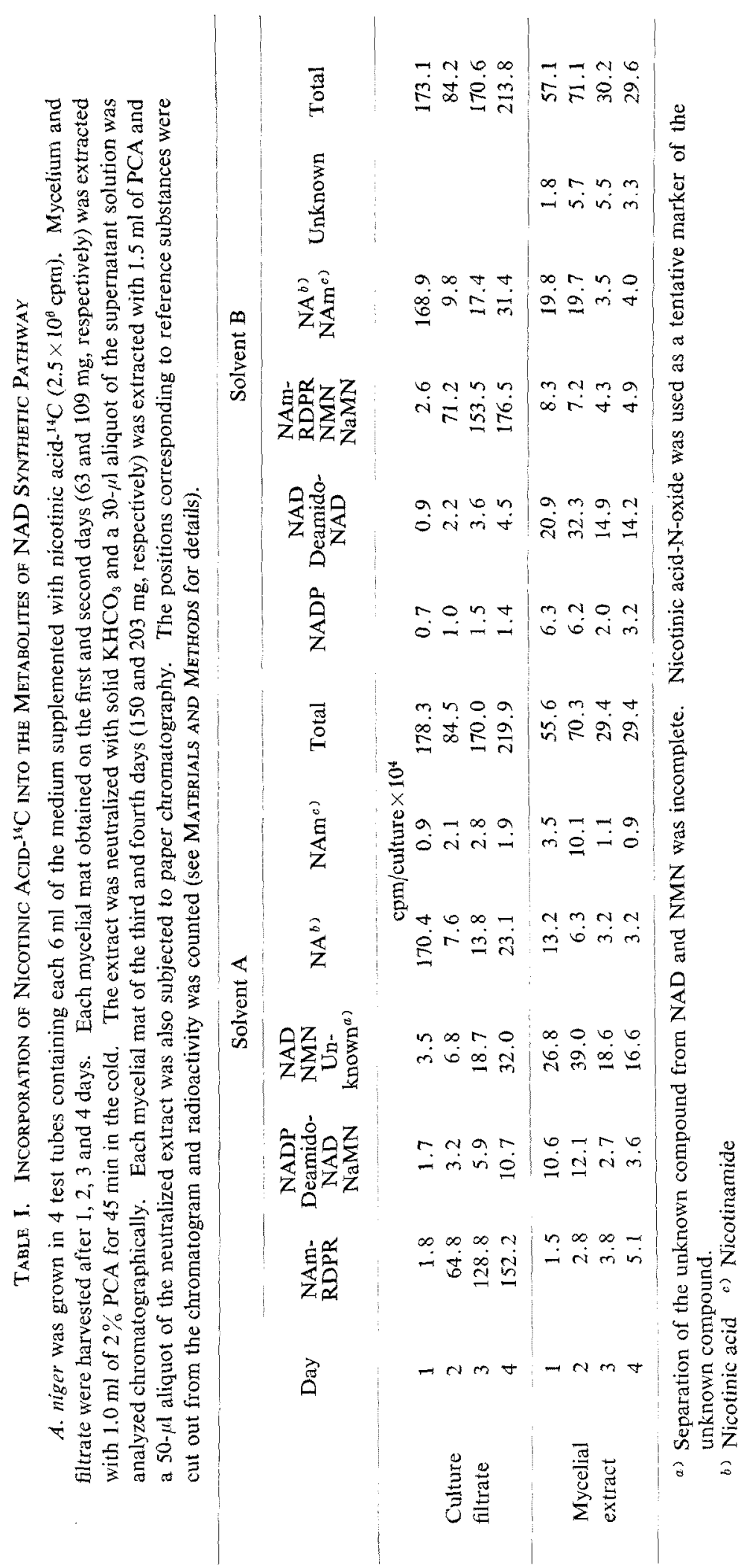




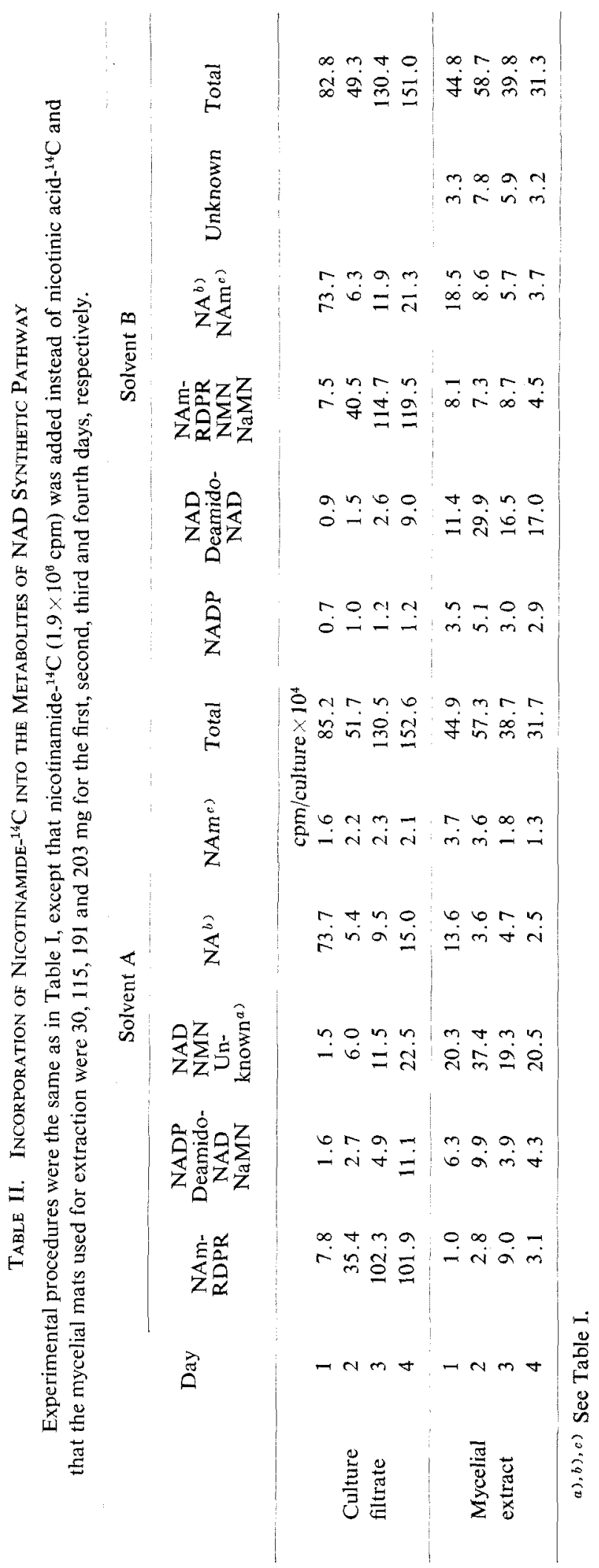


of NAD cycle in this organism. An unknown radioactive substance, other than those intermediates, was detected on the chromatogram. This substance has a very similar $R f$ value to that of nicotinic acid- $\mathrm{N}$-oxide and developed into the areas of peaks 4,4 and 2 in solvents $A$, $B$ and $C$, respectively. The amounts of radioactivity in these areas were considerably high. However, no clear evidence which indicates the nature of this compound has been obtained. In the present experiments nicotinic acid- $\mathrm{N}$-oxide was tentatively used as a reference marker of the unknown compound.

The labeling pattern of the nicotinamide $-{ }^{14} \mathrm{C}$ grown mycelium was similar to that of the nicotinic acid- ${ }^{14} \mathrm{C}$ grown mycelium (Fig. 5). This result indicates that nicotinamide is metabolized via a similar pathway to that in the case of nicotinic acid.

\section{Distribution of radioactivity in culture fltrate and mycelial extract}

Tables I and II show the change of labeling pattern of the intermediates as a function of culture time. Radioactive intermediates were excreted to the medium from mycelium with the prolonged cultural time; on the fourth day, about $85 \%$ and $80 \%$ of added nicotinic acid $-{ }^{14} \mathrm{C}$ and nicotinamide $-{ }^{14} \mathrm{C}$, respectively, were present in the medium. On the second and further days of culture, most of the radioactivity in the medium was found in the NAmRDPR fraction. When nicotinic acid was added as precursor, 77, 76 and $69 \%$ of the radioactivity in the medium were detected on NAmRDPR on the second, third and fourth day, respectively. Radioactivity on other intermediates excreted into the medium was low comparing with that on NAmRDPR.

The labeling pattern of the mycelial extract was quite different from that of the culture filtrate. Incorporation of precursors into the intermediates in mycelium reached a maximum on the second day. In the young culture (the first and second day), radioactivity was found mainly in the fraction containing NAD and that on NAD reached a maximum on the decond day. NADP synthesis was ob- served, and the amount of radioactivity on NADP decreased as increasing the culture duration. Radioactivity on NAmRDPR was considerably low comparing with that on NAmRDPR in the culture filtrate. The amounts of NaMN and deamido-NAD decreased with an increase of culture duration.

Radioactivity was also found on an unknown compound, which had been separated from other intermediates only in solvent B, and the formation of this compound reached a maximum on the second day in both of nicotinic acid- and nicotinamide- ${ }^{14} \mathrm{C}$ supplemented cultures.

\section{DISCUSSION}

As reported previously ${ }^{8}$ degradation of NAD at the adenine-ribose bond with an $A$. niger enzyme gives a new pyridine nucleotide, NAmRDPR. Questions have been raised if this compound is formed during the cultivation of the mold, and if this compound is a metabolite of the NAD pathways. The present experiment using tracers clearly demonstrates that NAmRDPR is produced from the precursors, nicotinic acid and nicotinamide, by growing cells of $A$. niger (Figs. 1 and 2). Additional experiments indicates that NAmRDPR is a terminal metabolite of the NAD pathway (Tables I and II). First, NAmRDPR was excreted into the medium and the level of radioactive NAmRDPR in the medium was much higher than that in the mycelium. Second, maximal accumulation of NAD proceeded that of NAmRDPR; production of NAD reached a maximum on the second day, whereas the maximal excretion of NAmRDPR was observed on the third or fourth day. Third, the amount of NAmRDPR rised with an increase of cultural duration and the level of NAmRDPR was maintained after the production had reached maximum. Most probably, a nucleosidase-type enzyme which attacks the adenine-ribose bond of NAD functions in producing NAmRDPR from NAD.

The present experiment suggests that NAD is 
;ynthesized via the Preiss-Handler pathway in 4. niger, as a high level of nicotinic acid was letected after short time of culture in the nedium supplemented with nicotinamide $-{ }^{14} \mathrm{C}$ Table II). This indicates the presence of leamidation reaction of nicotinamide, which $s$ the first step of the pathway. Formation of radioactive $\mathrm{NaMN}$ and deamido-NAD, alhough the contents of these intermediates were small (Figs. 3 and 4, Tables I and II), also ndicates the function of this pathway. NADase reported for $A$. niger by Sarma et al. ${ }^{3)}$ $s$ thought to participate in the NAD metaboli$; m$ in a strain of AKU 3302. As reported in he previous paper ${ }^{8 /}$ the cell-free extract of this itrain liberated nicotinamide from NAD in a ЈH range higher than 5.0. Therefore, radioictive nicotinamide which was accumulated naximally at the second day in the mycelium srown in nicotinic acid (Table $\mathrm{I}$ ) is thought to se formed from NAD by the action of NAJase. Temporarily accumulated nicotinamide was metabolized further via the Preiss-Handler athway and its radioactivity level dropped. The amount of nicotinic acid excreted into the sulture medium increased gradually after the ;econd day as an increase of culture time, and :his compound was most probably formed with he deamidation of nicotinamide. Possible ormation of nicotinamide and nicotinic acid rom NAmRDPR was not clearly indicated in he present experiments. Proposed pathway of NAD metabolism in $A$. niger is shown in Fig. 6.

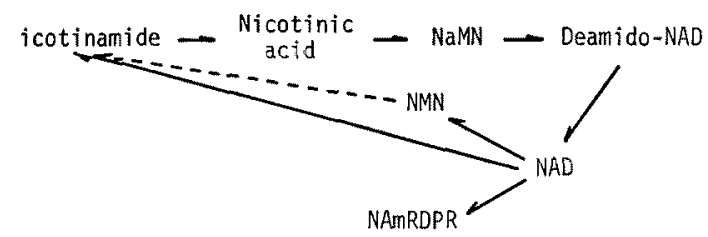

FIG. 6. A Proposed Pathway of NAD and NAmRDPR Syntheses in A. niger.

No clear evidence for the existence of niotinamide-dependent pathway, through which licotinamide is incorporated into NAD via JMN, was obtained. A small amount of
NMN found in the mycelial extract (Figs. 3 and 4, Tables I and II) was regarded as being formed from NAD instead of being directly formed from nicotinamide.

The present experiment shows that radioactivity from either nicotinic acid- or nicotinamide $-{ }^{14} \mathrm{C}$ was also incorporated into an unknown compound (Figs. 3 and 4). This compound exhibited similar behaviors to that of nicotinic acid- $\mathrm{N}$-oxide in paper chromatography examined with three different developing solvents. However, in a preliminary experiment, the eluting position of this compound in column chromatography with Dowex 1 (formate form) was shown to be different from that of nicotinic acid-N-oxide. The nature of this compound has not been welldefined so far.

Acknowledgement. The author wishes to thank Professors K. Ogata and T. Tochikura of Kyoto University for their useful advices and suggestions. Thanks are also due to Professor K. Yamanaka of Kagawa University for his advice and encouragement throughout this work.

\section{REFERENCES}

1) J. Preiss and P. Handler, J. Biol. Chem., 233, 493 (1958).

2) S. Chaykin, Ann. Rev. Biochem., 36, 149 (1967).

3) D. S. R. Sarma, S. Rajalakshmi and P. S. Sarma, Biochim. Biophys. Acta, 81, 311 (1964).

4) R. K. Gholson, J. Vitaminol., 14, 114 (1968).

5) J. Keller, M. Liersch and H. Grunicke, Eur. J. Biochem., 22, 263 (1971).

6) M. Kuwahara and S. Chaykin, J. Biol. Chem., 248, 5095 (1973).

7) E. Ohtsu, A. Ichiyama, Y. Nishizuka and $O$. Hayaishi, Biochem. Biophys. Res. Comm., 29, 635 (1967).

8) M. Kuwahara and M. Tsukamoto, Agr. Biol. Chem., 39, 1975 (1975).

9) T. Hondo, M. Ikeda, A. Andreoli, Y. Nishizuka and O. Hayaishi, Biochim. Biophys. Acta, 89, 549 (1964).

10) Y. Nishizuka and O. Hayaishi, J. Biol. Chem., 238, 3369 (1963).

11) Y. Hagino, S. J. Lau, C. Y. Ng and L. M. Henderson, ibid., 243, 4980 (1968).

12) S. Chaykin, M. Dagani, L. Johnson and $\mathbf{M}$. Samli, ibid., 240, 932 (1965). 